\title{
Preliminary results from attenuation correction for MCNP-generated PET image
}

\begin{abstract}
This paper presents the preliminary results of PET system simulation using Monte Carlo code. We also present the implementation of attenuation correction for MCNP-generated PET image. Using MCNP5 we constructed a data base for a uniform cylindrical source. The data obtained from the simulation were then used for PET image reconstruction. During the image reconstruction, calculated attenuation correction method was implemented to the PET raw data. This method was chosen due to the fact that our study involved homogeneous and simple geometry phantom.
\end{abstract}

Keyword: Attenuation correction; Monte carlo; PET 